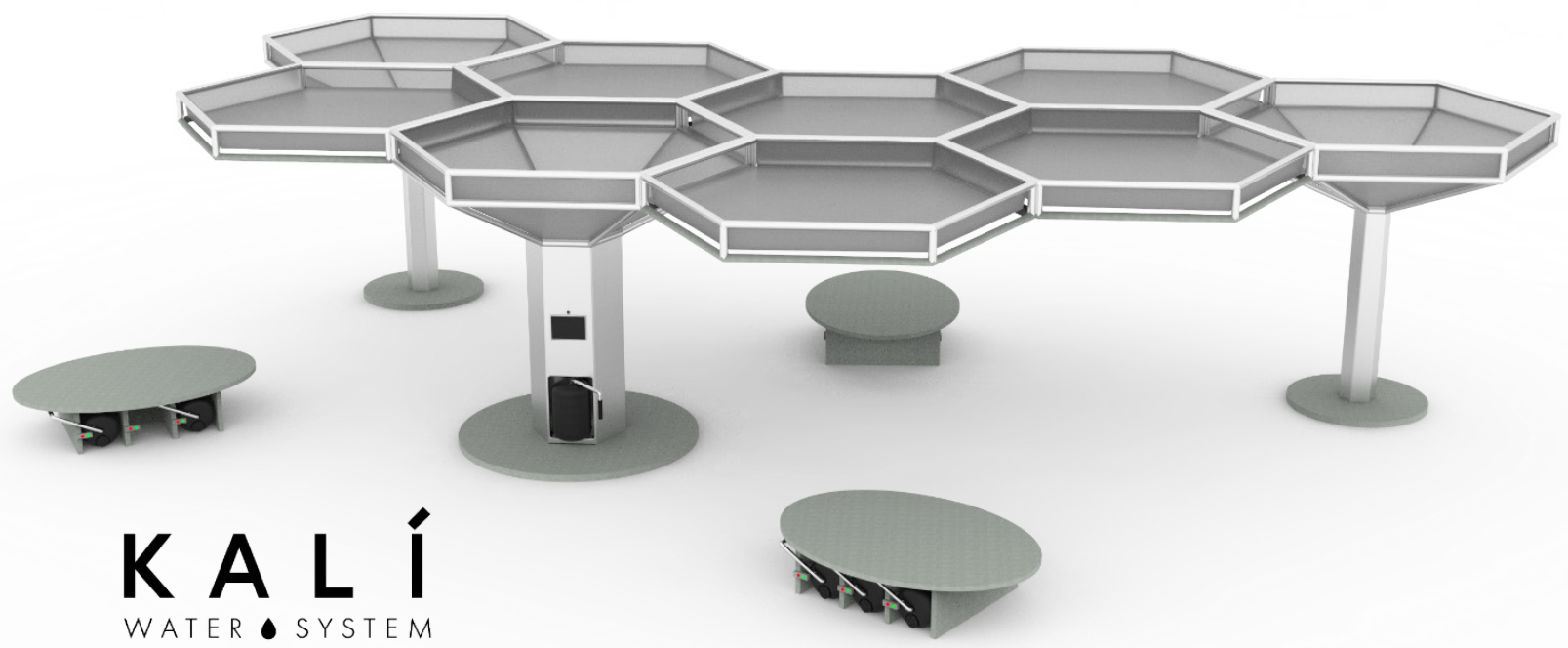

\title{
Diseño de sistema inteligente para la distribución equitativa de agua en la GAM, Costa Rica
}

Intelligent system design for equitable water distribution in GAM, Costa Rica

Kristhian Solano-Camacho ${ }^{1}$, Violeta Morice-Fernández ${ }^{2}$

K. Solano-Camacho, V. Morice-Fernández, “Diseño de sistema inteligente para la distribución equitativa de agua en la GAM, Costa Rica", IDI+, vol. 3, no 2, Ene., pp. 4-15, 2021.

doi) https://www.doi.org/ 10.18845/ridip.v3i2.5562

Fecha de recepción: 5 de agosto de 2020 Fecha de aprobación: 29 de setiembre de 2020

1. Kristhian Solano-Camacho Estudiante de Ingeniería en Diseño Industrial Instituto Tecnológico de Costa Rica Cartago, Costa Rica krisc1999@estudiantec.cr (D) 0000-0003-2077-7477

2. Violeta Morice-Fernández Estudiante de Ingeniería en Diseño Industrial Instituto Tecnológico de Costa Rica Cartago, Costa Rica vmorice@estudiantec.cr

(D) 0000-0002-9675-6579 


\section{Resumen}

Año tras año, la escasez de agua crece afectando a un alto porcentaje de la población mundial. En Costa Rica y Latinoamérica, la urbanización aumenta aceleradamente sin tener una adecuada infraestructura de servicios públicos. Durante la época seca, el acceso al recurso hídrico se ve afectado y los mecanismos de abastecimiento utilizados, como camiones cisterna, no son accesibles para toda la población; por esto, se definió como problema una mala sistematización en la distribución del agua.

Este artículo muestra el proceso de diseño llevado a cabo para desarrollar un sistema de productos inteligentes que mejoren la distribución del agua, desde su captación hasta su transporte a casa. La funcionalidad se determinó sintetizando requerimientos definidos a partir de necesidades mostradas por amas de casa, jóvenes estudiantes y operarios del sistema de acueductos.

La morfología desarrollada respondió al concepto "integración biónica” que buscaba la manera de crear una convivencia integral del producto en su entorno y hacer cumplir sus funciones y estética a partir de propiedades biónicas. La propuesta final contempló el uso de agua pluvial como principal insumo, una configuración hexagonal como medio de estructura y autonomía por medio de procesos tecnológicos.

El diseño de productos inteligentes debe ser utilizado como herramienta de innovación en la búsqueda de soluciones a problemáticas sociales, contar con evaluaciones de impacto desde corto hasta largo plazo y garantizar una mejor calidad de vida para sus usuarios.

\section{Palabras clave}

Diseño social; diseño industrial; distribución equitativa del agua; Latinoamérica; biónica.

\section{Abstract}

Year in year out, lack of water increases affecting a high percentage of the world's population. In Costa Rica and Latin America, urbanization grows rapidly without an adequate public services infrastructure. During the dry season, the access to the hydric resource gets affected and the supplying mechanisms used, like tank trucks, are not available for all the people; for this reason, the problem was defined as a bad systematization of water distribution.

This article shows the design process executed to develop a smart product system that improves water distribution, from its harvesting until home transportation. Functionality was determined by requirements defined from needs shown by housewives, young students, and aqueduct system operators. 
The developed morphology responds to the concept "bionic integration" that looks for the way to create an integral coexistence of the product in its environment and achieve its functions and aesthetics from bionic properties. The final design contemplated rain water as main intake, an hexagonal configuration as a structural base and autonomy due to technological processes.

Smart products design should be used as a tool to innovate in social problems, evaluate impact front short to long terms and guarantee a better users wellbeing.

\section{Keywords}

Social design; industrial design; equal water distribution; Latin America; bionics.

\section{Introducción}

El reto propuesto fue diseñar un producto inteligente para una ciudad inteligente. Según el Ministerio de Ciencia, Tecnología y Telecomunicaciones (MICITT), existen seis componentes con sus respectivos indicadores para calificar una ciudad como una inteligente: inteligencia en gobierno, en educación, en infraestructuras y redes, inteligencia en economía, en convivencia social e inteligencia en ambiente y energía [1]. A pesar de ser una lista completa, hace falta algo esencial: acceso al agua. Una buena distribución y acceso de este recurso garantiza la reducción y propagación de enfermedades relacionadas al saneamiento y la salud, así como pone un freno a la desigualdad social e impulsa la economía.

Se llega a la conclusión de que el acceso al agua es una de las bases esenciales para construir una ciudad inteligente, como parte de un país en vías de desarrollo, los ingenieros en diseño industrial deben apuntar al mejoramiento de la sociedad, impulsando a las zonas con mayores necesidades.

Actualmente, el $40 \%$ de la población mundial presenta escasez de agua y se estima que esta cifra aumente [2]. Para el año 2050, Latinoamérica se convertirá en la segunda región más urbanizada del mundo, ubicando hasta 100 millones de personas en asentamientos irregulares bajo una deficiente infraestructura de servicios públicos [3]. Por su parte, Centroamérica cuenta en general con una elevada disponibilidad hídrica, pero el agua no llega a toda la población o lo hace sin ser de calidad [4], lo que evidencia que la gestión del recurso en la región es apenas incipiente.

En los primeros meses del año 2020, más de 320.000 personas han sido afectadas por falta de agua en la Gran Área Metropolitana (GAM) debido al déficit de lluvias por el que el país ha estado pasando. En una entrevista realizada a Roy Barboza, subgerente del sistema GAM del Instituto Costarricense de Acueductos y Alcantarillados (AyA), comenta que es difícil para esta entidad definir con claridad las horas en las que el agua no estará disponible, por múltiples razones, tales como el estrés y angustia de los habitantes, quienes como consecuencia guardan 
agua en cualquier objeto a su disposición con tal de no verse afectados por el corte. Esto causa que los tanques del AyA se vacíen más rápido de lo previsto, provocando más horas sin este recurso y dificultando las estimaciones de los operarios del AyA con respecto a las horas de cortes. Estas situaciones ponen en evidencia la falta de comunicación y organización entre los habitantes y sus respectivas municipalidades [5].

La problemática es una mala sistematización en la distribución del agua. Por esto se propone el diseño de un sistema de productos que faciliten la distribución del agua, así como el acceso continuo y transporte en las comunidades de la GAM; el propósito es asegurar un acceso equitativo a este recurso esencial para la vida y el desarrollo de una ciudad inteligente.

Como resultado se obtuvo un sistema de productos compuesto por una estación central, encargada de la captación, purificación, almacenamiento, disposición del agua, entre otras, y un dispositivo de transporte que permitirá a los usuarios llevar el agua de la estación a sus casas de una manera sencilla. La participación activa de los usuarios incluso de manera virtual toma gran importancia al realizar el proyecto y se percibe el gran impacto que pueden tener los ingenieros en diseño industrial al involucrarse en proyectos de temática social.

\section{Método}

Para el contexto vivido en el desarrollo del proyecto (primer semestre del 2020), el mundo se encontraba en período de cuarentena debido a la pandemia del COVID-19, por lo que la metodología e instrumentos implementados en adelante fueron completamente virtuales, garantizando la salud de los estudiantes y posibles usuarios del producto.

El proceso de diseño de Kalí constó de cinco etapas: conceptualización de la idea, definición de la funcionalidad, definición de la forma, detallado de la manufactura y la simulación del diseño.

\section{Conceptualización de la idea}

El proyecto inició con una investigación del contexto actual y futuro sobre el acceso al agua en el país y el mundo, además, se definió como entorno de uso del producto: zonas públicas del Gran Área Metropolitana. Para tener un mayor conocimiento de la situación vivida en el área geográfica elegida, se realizaron entrevistas a personas ubicadas en los perfiles de usuario previamente definidos. La síntesis de esta información, sumada a la descrita anteriormente, facilitó la definición del problema, este fue planteado a partir de la herramienta árbol de problemas $^{1}$, donde se derivan causas y consecuencias para buscar la raíz y concretar el problema. Con la información obtenida hasta el momento, se plantearon las necesidades y requerimientos que el producto debería satisfacer buscando solucionar el problema indicado. Se

1 Árbol de problemas: es una herramienta participativa que se utiliza para identificar los problemas principales con sus respectivas causas y efectos. Esto permite planificar y definir objetivos claros. 
definió un segmento de mercado organizacional donde se encuentran instituciones encargadas de la reserva y distribución del recurso hídrico.

El conocimiento de productos que se encuentren actualmente en el mercado o en etapas conceptuales es de gran valor para evaluar ventajas y desventajas que tienen diseños ya ejecutados para solucionar las necesidades previstas. Se realizó un análisis de lo existente ${ }^{2}$ para concluir sobre los temas de regulación del suministro de agua, su transporte y métodos de captación del recurso, además, tomando en cuenta que sería un producto para espacios públicos, se optó por investigar desarrollos de mobiliario urbano. Como cierre de la etapa, se formuló una lista de especificaciones, que describe las características indispensables o deseables del producto basadas en la definición de necesidades y requerimientos de los usuarios; estos fueron validados por posibles usuarios, lo que permitió jerarquizar las diferentes funciones para toma de decisiones a futuro.

\section{Definición de la funcionalidad}

Una vez jerarquizados los requerimientos, se procedió a elaborar un árbol de funciones. Se determinó una función principal para el sistema y mediante el modelo FAST $^{3}$, se derivaron las funciones prácticas e inteligentes; entre las que destacan: captación de agua, purificación de agua, monitoreo de estado y facilidad de uso. Se eleva un énfasis en las inteligentes dado el objetivo del proyecto, y se realizó una exploración morfológica sobre las posibles soluciones para las mismas. Con la ayuda del análisis de referenciales se evaluaron aspectos funcionales, de interacción y manufactura.

En el área funcional, se buscaba definir los componentes electrónicos que ejecutarán las funciones inteligentes de los productos mediante una matriz multicriterio, que contrasta los componentes y sus especificaciones técnicas para, posteriormente, hacer la elección de los óptimos. La interacción tenía como fin explorar distintos métodos en los que los usuarios se relacionarían con el producto y este con su entorno. Como tercer punto en esta etapa, se generó un diagrama de flujo para describir el funcionamiento de los productos. Este permite evaluar de manera eficiente si se cuenta con todos los componentes necesarios para realizar cada una de las funciones.

Se definió cada uno de los productos como un sistema, detallando sus subsistemas, componentes y partes que lo componen.

2 Análisis de lo existente: se utiliza con el objetivo de conocer los productos existentes que satisfacen la misma necesidad, es posible también utilizar productos similares. Esto con el fin de analizar las diferentes funciones, características de diseño y soluciones propuestas por otros productos.

3 Análisis Funcional de Sistemas Técnicos: muestra una disposición horizontal denominada dimensión "Cómo/Porqué". Estas son las preguntas que se emplean para estructurar la lógica del sistema que constituye el producto desde el cómo se realiza y el porqué se realiza. 


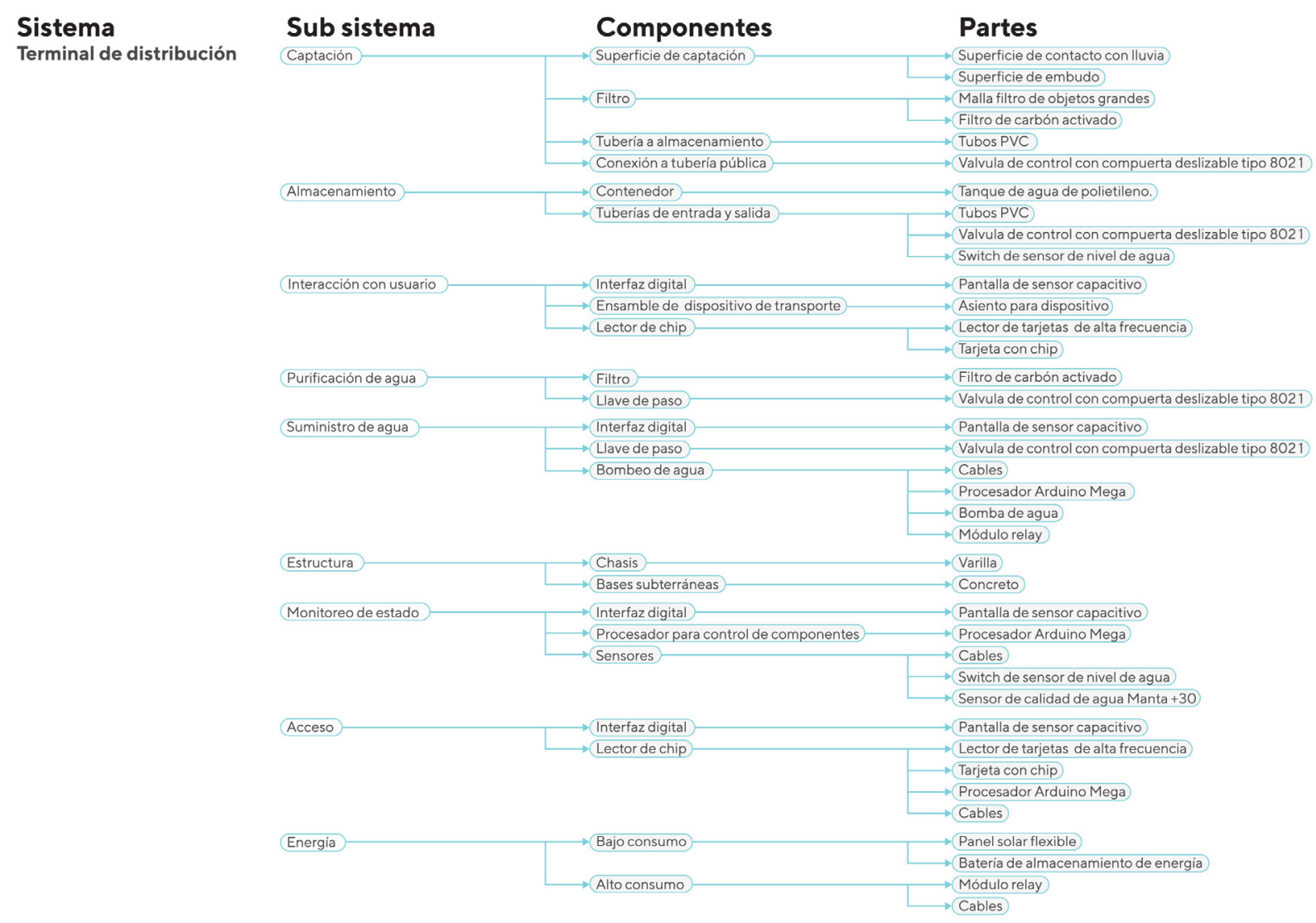

Figura 1. Estación central como sistema

Fuente. Elaboración propia, 2020

Es importante destacar que algunos de los componentes señalados en la figura 1 fueron modificados en etapas posteriores, esto debido a la constante iteración que debe existir en el proceso de diseño de cualquier producto para evaluar la mejor manera de hacer cumplir las funciones. Para iniciar las propuestas, se dibujaron los primeros bocetos, que transmiten la idea volumétrica del sistema de productos que se está desarrollando y su relación con la silueta humana. Nuevamente se realizó una validación, en este caso de las funciones inteligentes planteadas. También se abrió el espacio para recibir retroalimentación de los usuarios.

\section{Definición de la forma}

Esta etapa se inició definiendo el concepto de diseño, a este se le deben sumar variables que explican de qué manera se cumplía este en la propuesta. Emitir los objetivos que daban respuesta a la problemática definida al inicio del artículo, asimismo, contemplar los alcances que tendrá el proyecto. Se eligieron las "WH questions" para describir de una forma sintética todos los aspectos del sistema de productos (qué, porqué, para qué, dónde, cómo, para quién). 
La ubicación de los componentes donde habrá interacción se definió basado en un análisis antropométrico parte del análisis ergonómico, donde también evaluó la disposición cognitiva de las interfaces digitales.

Para definir la perceptualidad del producto, se canalizó un cuadrante de un vocabulario visual que posteriormente fue definido como frase semántica para realizar un moodboard ${ }^{4}$ y concretar el look and feel del sistema. Con la perceptualidad lista, se diseñaron alternativas a nivel de boceto de alta calidad, contemplando volumen, forma y estética. Con estas se realizó una evaluación de requerimientos para elegir la propuesta final y, finalmente, se detalló la elección.
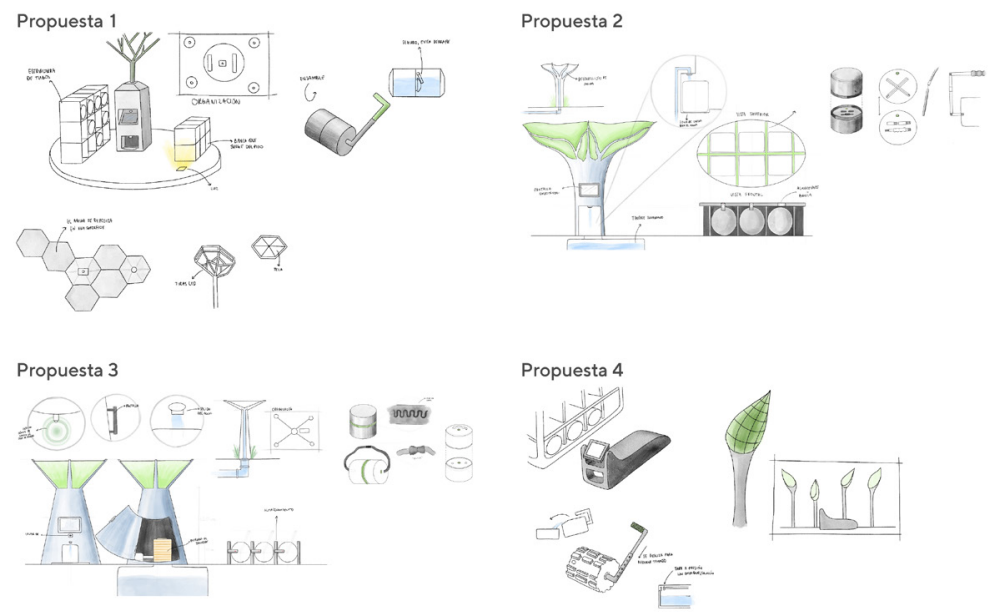

Figura 2. Diseño de alternativas

Fuente. Elaboración propia, 2020

\section{Detallando la manufactura}

En esta cuarta etapa se describieron las funciones que cumple el sistema, tanto prácticas como inteligentes, se continuó con una descripción de la arquitectura planteada mostrando todas las partes que componen el sistema. Se concretaron los materiales y se realizó una justificación de los mismos. Se construyeron los planos técnicos de cada una de las partes del producto que deban ser manufacturadas y se compuso la lista de los componentes normalizados y estandarizados que estarían presentes en el sistema para asegurar su funcionamiento. El uso del producto estuvo sintetizado en un manual de usuario que incluyó instalación, mantenimiento y el uso de cada uno de los productos. Finalmente se describió el proceso de manufactura de las piezas que componen el sistema y se realizó una proyección de los costos de producción de una instalación del producto.

4 Moodboard: consta de una combinación de imágenes, texturas, colores, materiales y conceptos que describen emociones y sentimientos que se desean en el diseño por proponer. 


\section{Simulación del diseño}

En esta última etapa se realizó una síntesis de todo el trabajo, en la cual se incluyó: el contexto, los usuarios, el concepto, el funcionamiento, la configuración y la interacción; esto con el fin de proporcionar una idea clara de los objetivos del proyecto, así como los resultados finales. Se realizaron renders de alta calidad y videos simulados, estos permitieron demostrar en detalle las funciones y las interacciones presentes en el producto.

\section{Discusión de resultados}

El concepto definido en la figura 3 muestra el punto de partida para los desarrollos formales que se contemplen, además de la unión entre la funcionalidad y la estética. Durante la realización del diagrama de funciones, se notó que, para cumplir a cabalidad los requerimientos, era necesario crear un sistema de productos; se establece la estación central, dispositivos de transporte y módulos de almacenamiento, este último no fue contemplado en las últimas etapas, debido a los alcances del proyecto.
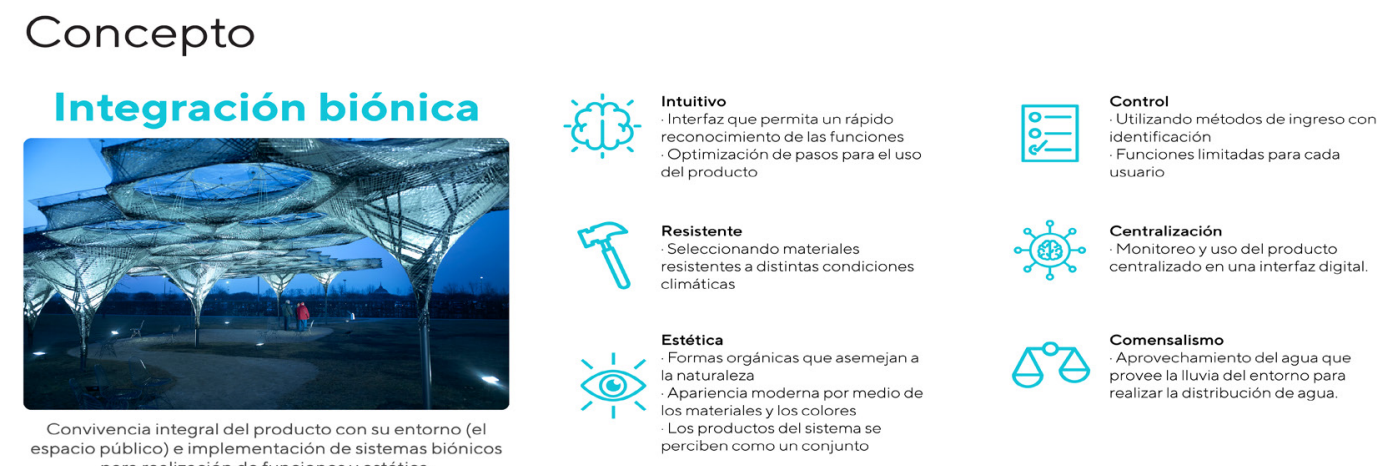

Figura 3. Concepto y variables de diseño

Fuente. Elaboración propia, 2020

La estética del producto se definió a partir de la frase "Modernidad natural", donde se buscaba que el sistema se integrara por su morfología simple al entorno y brindara a los usuarios el sentimiento de poder interactuar con él. La cromática se ve abarcada completamente por el tono blanco y presencia de grises, también se utilizó la forma hexagonal similar a la de panales de abeja para suplir ese sentimiento natural, todas las aristas son redondeadas para transmitir formas orgánicas. 


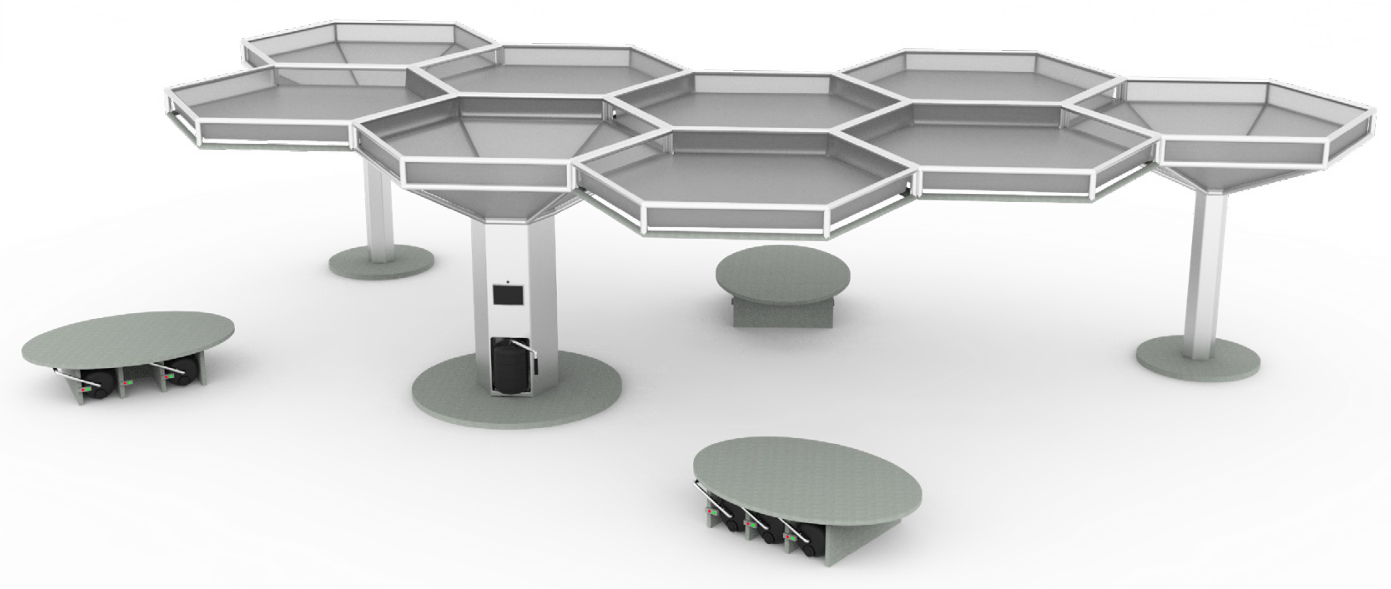

Figura 4. Sistema Kalí

Fuente. Elaboración propia, 2020

Entre los aspectos funcionales de la estación central, se contempla el aprovechamiento pluvial para obtener el agua con superficies de captación de $9 \mathrm{~m}^{2}$, se aprovechó la funcionalidad estructural y morfológica de los hexágonos para generar estas superficies y crear módulos para adaptar la instalación en distintos espacios. Si el almacenamiento se encuentra lleno, permite brindar $120 \mathrm{~L}$ de agua diariamente a 60 familias, además, el producto puede ser utilizado durante cualquier hora del día para garantizar disponibilidad de emergencia de agua potable a cualquier usuario.

Se implementa un sistema de doble filtrado por medio de carbono comprimido para propiciar la potabilidad del agua, este garantiza la remoción tanto de residuos orgánicos como partículas sólidas inertes, además, para facilitar el monitoreo de la potabilidad del agua, se cuenta con una multiprobeta Manta 40+ que realiza muestreos de agua con el fin de evaluar presencia de organismos, nivel de $\mathrm{pH}$ y oxígeno, entre otros, para definir su potabilidad. Cuenta con una interfaz táctil y un lector QR que restringirá el acceso a los usuarios y las distintas funciones que se pueden realizar desde esta pantalla.

La estación centraliza sus funciones con un procesador Arduino UNO WiFi especializado para sistemas de loT, encargado de tomar decisiones y generar las respuestas necesarias dependiendo del estado e información brindada por los sensores incorporados. Se cuenta con una boya en el tanque de almacenamiento que indicará al procesador cuáles válvulas deben estar abiertas para habilitar o cerrar el paso de agua de distintas fuentes. En caso de perderse la comunicación entre algún sensor y el procesador, este emitirá una notificación para alertar la falla al operador que realice mantenimiento, de esta manera, se fortalece el cumplimiento del objetivo de simplificar el análisis de estado de los componentes del producto. 


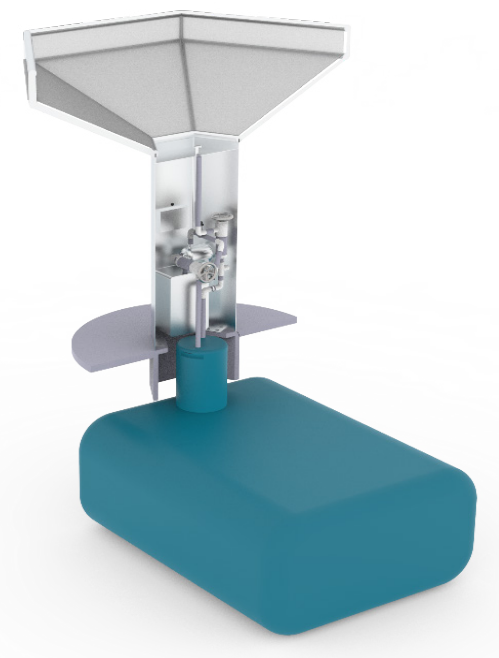

Figura 5. Corte de estación central

Fuente. Elaboración propia, 2020

El dispositivo de transporte permitirá llevar el agua de la estación a las casas de los usuarios; cuenta con una capacidad de volumen contenido de $60 \mathrm{~L}$, así como un agarre con largo ajustable para adaptarse al usuario y mejorar las condiciones de empuje y halado del producto. El producto será destinado a áreas delimitadas, por lo que contará con un módulo GPS que avisará al usuario mediante un zumbador, en caso de estar saliendo de este límite. La ubicación también será transmitida al procesador ubicado en la estación central para realizar análisis de datos. El procesador Arduino Mini loT y demás componentes están potenciados por una batería incorporada en el producto, para facilitar la carga de esta, se propone el uso de un módulo de carga inalámbrica.

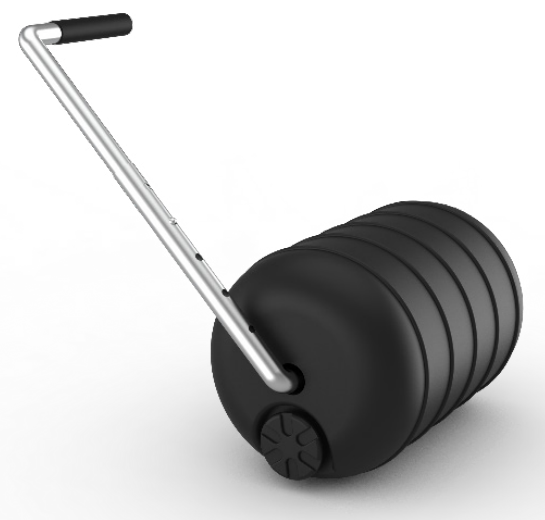

Figura 6. Dispositivo de transporte

Fuente. Elaboración propia, 2020 
Los procesos de manufactura que destacan en la estación central son el troquelado, doblado y soldado de piezas, repitiendo este proceso para el dispositivo de transporte y agregando el rotomoldeo para el tanque de transporte de agua.

Los sistemas de funcionamiento no fueron probados por los motivos de virtualización mencionados al inicio del artículo, sin embargo, basados en las fichas técnicas de cada uno de los componentes elegidos, se cumpliría a cabalidad cada una de sus funciones.

Los productos logran sistematizar el acceso al agua de una manera equitativa y segura, en la que se busca afianzar el sentimiento de comunidad y conciencia social, con el fin de mejorar la calidad de vida de los habitantes de la zona en períodos de emergencia.

\section{Conclusiones}

La validación, retroalimentación y recomendaciones de los usuarios son importantes durante todas las etapas de diseño, ya que ayudan a consolidar las variables de diseño planteadas y reducen la posibilidad de cometer errores al generar las alternativas de diseño.

Conociendo el impacto que podría tener una solución como Kalí en el mercado, se perciben los amplios beneficios que tiene la aplicación del diseño industrial en la solución de problemáticas sociales. En este proyecto específicamente, la implementación de un sistema inteligente para resolver el acceso equitativo al agua lograría combatir la brecha social existente en la infraestructura de servicios públicos, que continúa en aumento en las zonas urbanas de Latinoamérica.

Durante las etapas investigativas, se identificaron otros problemas sociales que claramente perjudicaban el que se decidió atacar. La falta de educación en temas de uso eficiente del recurso hídrico, desinterés comunal y la deficiente comunicación entre instituciones y pobladores, son algunos ejemplos de problemas percibidos que podrían ser resueltos bajo la ejecución de un plan de diseño estratégico que se apoye en los objetivos 6,10 y 11 de desarrollo sostenible de la ONU, los cuales garantizan respectivamente: agua limpia y saneamiento, reducción de desigualdades y comunidades y ciudades sostenibles [6].

Como concepto de diseño se utilizó el término "biónica" que comúnmente se contempla desde lo estético, sin embargo, para este sistema de productos, se especificó más desde el punto de vista funcional, estructural y de relación con el entorno.

Finalmente, se recalca en la iteración continua, un concepto que permite adaptar el sistema conforme se avanza en el proceso de diseño; volver a etapas previas permite evaluar si lo planteado sigue funcionando, se encontró una mejor manera de hacerlo o, como en este caso, se deben cambiar componentes o materiales para cumplir satisfactoriamente las funciones. 


\section{Reconocimiento}

Este proyecto no se habría logrado concretar de no ser por la guía del profesor IDI. Luis Carlos Araya Rojas, quien logró adaptar pedagógicamente el curso: Diseño $\mathrm{V}$, a un contexto pandémico, sin que se vieran totalmente perjudicados los alcances del proyecto e interés de los estudiantes. Le agradecemos por su alto desempeño docente, en el que mantuvo una postura de respeto y cercanía en cada una de las etapas.

\section{Referencias}

[1] Ministerio de Ciencia, Tecnología y Telecomunicaciones Costa Rica. "Índice de Ciudades Inteligentes, 2016" Disponible en: https://www.micit.go.cr/sites/default/files/informe-ici2016-pdf.pdf

[2] Gobierno de la republica de Costa Rica y ONU (2020). Objetivo 6 Garantizar la disponibilidad de agua y su gestión sostenible y el saneamiento para todos. Disponible: http://ods.cr/objetivo/objetivo-6.

[3] D. Arguedas, "Las ciudades, protagonistas ante cambio climático en América Latina," Inter Press Service, pp. n/a, 2017. Disponible en: https://search-proquest-com.ezproxy. itcr.ac.cr/docview/1929685311?accountid=27651.

[4] G. Ferullo y F. Agrana, "En Centroamérica la gestión del agua es un asunto que apenas está comenzado: CENTROAMÉRICA DÍA AGUA," EFE News Service, pp. n/a, 2019. Disponible en: https://search-proquest-com.ezproxy.itcr.ac.cr/ docview/2195272345?accountid=27651.

[5] K. Zúñiga, "Más de 67 mil personas afectadas por falta de agua," 2020. Disponible en:https://www.multimedios.cr/nacional/mas-de-67-mil-personas-afectadas-por-faltade-agua.

[6] Organización de las Naciones Unidas. "Objetivos de Desarrollo Sostenible”. Disponible en: https://www.un.org/sustainabledevelopment/es/objetivos-de-desarrollo-sostenible/ 